years; and the whole source of confusion lies in the failure of those responsible for the British Association Reports of the time to insert the author's initials - the Allmans having been ap. "Darently referred to indifferently as merely "Professor" and

While thus my precise statement concerning George James Allman's claim to distinction which is involved must be dissociated from his memory, perusal of his published writings still justifies us in regarding him as a scientific botanist of renown.

It has been pointed out to me that my reference to the late Beete-Jukes might be interpreted to mean that he was a professor in Trinity College, Dublin ; and, in event of this possibility, I would remark that no such idea was intended. George James Allman was appointed professor of botany in Dublin University, in succession to William Allman, in 1844 ; resigning the appointment in I856. Joseph Beete-Jukes became director of the Irish branch of the Geological Survey in 1850; and during the whole period of George Allman's occupancy of the Dublin botanical chair, the late Samuel Haughton was professor of geology. The allusion to the late Beete-Jukes was introduced into my notes by the kindness of a relative of the late George James Allman, and it is inaccurate as concerning the word "Professor," as I now find to be also the case with the word "Regius" as applied to the Dublin chair itself (which I owe to the obituary notice in the Times of November 28, I898). "Professor" (line 19) and "Regius" (line 18) must accordingly be deleted from my article by those who would make further use of it ; and I would remark that by "Grumera" (column 4), Gunnera is meant.

My best thanks are due to Mr. Britten, Mr. Griffith, and Prof. Percival Wright, for friendly assistance and advice in this interesting little bibliographic research, the limitations of which I appear to have by no means exhausted.

Royal College of Science, London, S.W., January 9.

Since the above was written, we have received the following from Prof. Allman.-ED.

When writing the above I did not recollect that my father - many years later-read at the meeting of the British Association in Dublin (1835) a paper "On the Mathematical Relations of the Forms of the Cells of Plants" (Brit. Assoc. Rep. 1835, part ii. p. 79). This paper is erroneously attributed to Dr. George James Allman in the Catalogue of Scientific Papers of the Royal Society of London.-G. J. A. January 16.

\section{The Density of the Matter composing the Kathode Rays.}

THE question of the size, charges and velocities of the carriers in the kathode rays has been made the subject of investigation by Prof. J. J. Thomson, Lenard, and others. I do not know whether it has been noticed that, by taking the values which have been obtained for the ratio of charge to mass, and for the velocity of the particles, in connection with the observed fact that a shaft of rays from a plane kathode retains its cylindrical form unaltered as it passes across the tube, we can arrive at a limiting value for the mass per unit volume of the matter composing the rays.

Take for simplicity a uniform circular shaft of charged particles travelling with velocity $u$. Let the mass per unit volume be $m$, the charge per unit volume $e$, the radius of the section $a$, and the velocity of light $v$. The shaft constitutes a current of strength $e u \cdot \pi a^{2}$. The magnetic force at the boundary is

$$
\mathrm{H}=\frac{4 \pi \quad e u \pi a^{2}}{2 \pi a}=2 \pi a e u .
$$

If we take unit volume at the boundary, it will be subject to an electro-magnetic force, inwards,

$$
=\mathrm{H} . e u=2 \pi a e^{2} u^{2} \text {. }
$$

The same unit volume will be acted upon further by an electrostatic repulsion outwards of amount $2 \pi a e^{2} v^{2}$ in electromagnetic units.

Hence the resultant force on it will be $2 \pi a e^{2}\left(v^{2}-u^{2}\right)$ outwards.

Now if $\rho$ be the radius of curvature of the outer boundary, we have

$$
\frac{m u^{2}}{\rho}=2 \pi a e^{2}\left(v^{2}-u^{2}\right),
$$

or the curvature is

$$
\frac{\mathbf{I}}{\boldsymbol{\rho}}=2 \pi a\left(\frac{e}{m}\right)^{2} m\left(\frac{v^{2}}{u^{2}}-\mathrm{I}\right)
$$

We know that this curvature is small.

Taking the numbers given by Lenard (Wied. Ann., 65, p. 504), we may put, roughly,

$$
\begin{aligned}
& \frac{e}{m}=6 \times 10^{6} \\
& \frac{v}{u}=4
\end{aligned}
$$

giving

$$
\begin{aligned}
\frac{\mathbf{I}}{\boldsymbol{\rho}} & =2 \pi a \cdot 36 \cdot 15 \cdot 10^{12} \cdot m \\
& =m a \times 3.4 \times 10^{15} .
\end{aligned}
$$

Therefore $m$ must be smaller than order $10^{-15}$, whereas the average density in the tube, that of air at the pressure of a fraction of a millimetre, is of the order $\mathrm{IO}^{-7}$.

If we do not suppose that $m$ and $e$ are constant, but take them as functions of the distance from the axis of the shaft, we arrive at the above limit for the average density.

Çueen's College, Belfast, January 9. $\quad$ W. B. MORTon.

\section{Attraction in a Spherical Hollow.}

Among the papers of the late Prof. Peter Alexander, of Anderson's Medical College, Glasgow, I find the enunciation of an interesting theorem in attraction. "The attraction on a particle of unit mass, in a spherical hollow in a sphere of uniform density, is at all points of the hollow parallel to the line joining the centres of the sphere and hollow, and is of constant magnitude equal to $\frac{4}{3} \pi c \sigma \kappa$. Where $c$ is the distance between the centres, $\sigma$ the density of the sphere and $\kappa$ the attraction of unit mass on unit mass at unit distance."

I venture to give the following informal proof. Let a be the centre of sphere which may be supposed to be indefinitely great, and $\mathrm{B}$ a particle at the centre of the spherical hollow. Then the attraction on $\mathrm{B}$ is towards $\mathrm{A}$, and is proportional to BA if the hollow be indefinitely small (see Dr. Tarleton's "Introduction to the Theory of Attraction," p. 13). But the removal of the spherical mass round $B$ as centre in no way alters the attraction on the particle $\mathrm{B}$. This proves the theorem for the central point. If the centre of the sphere were at $\mathrm{c}$ the attraction on $\mathrm{B}$ would then be $\mathrm{BC}$ in the same way. Let the

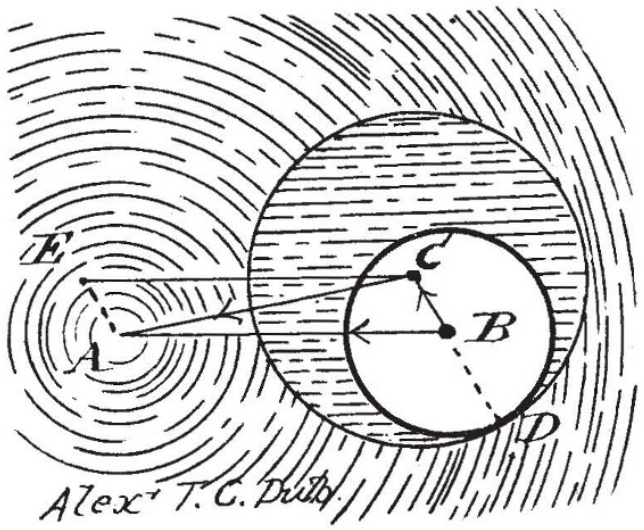

particle be now placed at $\mathrm{c}$ any point in the hollow. Produce CB to meet the hollow sphere at D. If the spherical hollow be enlarged so that $\mathrm{C}$ is its centre and $\mathrm{CD}$ its radius, the force of attraction on $\mathrm{C}$ will now be CA. Restoring the mass to the space between the new and the original hollows, subjects the particle at $\mathrm{c}$ to an additional force, equal and opposite to BC. Hence the force exercised on the particle at $\mathrm{c}$ in the original hollow is CE, which is parallel and equal to BA.

This furnishes a good example of the theorems (ibid., pp. 60 and 94), that if one or other the amount or direction of the attraction within unoccupied space be constant, then must both be so.

There is probably a formal rigid proof of his theorem among my brother's papers. He told me that some practical application might be made, by having the hollow just touching the

NO. I 525 , vOL. 59] 